\title{
The strategic sequelae of SARS-CoV-2 vaccination policies utilized by NATO and NATO-aligned countries, in the context of Combined-Arms Cyber Warfare.
}

James D. Sheehan

\begin{abstract}
Combined-Arms Cyber Warfare utilizes cyber-attack, sabotage of infrastructural elements and Electromagnetic Pulse (EMP) attack, alone or in combination with conventional military tactics, to conduct warfare against opposing military forces and the civilian populations and/or infrastructures which they defend ${ }_{(1)}$. Nuclear EMP attacks have been acknowledged to constitute a central component of the military doctrines, plans and exercises of the Combined-Arms Cyber Warfare doctrines of multiple nations (1). A Nuclear EMP attack consists of the detonation of thermonuclear weapons in a manner that minimizes the energy liberated in the form of blast (overpressure) and thermal effects, while ensuring an increased proportion of detonation energy is directed to the targeted area as prompt radiation emissions and an associated electrical field ${ }_{(1,2)}$. Significant overlap exists between the design and function of Nuclear EMP weapons and that of Enhanced Radiation Weapons (ERW), colloquially known as "Neutron bombs" $(1,2,3)$. ERW are also a class of fission-fusion devices specifically designed to limit the energy of detonation manifested as blast and thermal effects while maximizing the percentage of energetic yield released as prompt radiation, with a particular emphasis on gamma-neutron emissions $(2,3)$. Neutrons have been reported to be more biologically hazardous than ionizing photons on a per dose basis (7). Slow and Thermal neutrons have been reported to possess a greater relative biological effectiveness (RBE) for cell death, single-strand DNA breakage and double-strand DNA breakage, relative to an equivalent dose of gamma-radiation $(7,8)$. The full-length SARS-CoV2 spike glycoprotein has been reported to directly inhibit double-strand break (DSB) DNA repair in the absence of SARS-CoV-2 infection (9). Additionally, the spike glycoprotein of SARS-CoV-2 impairs the expansion of hematopoietic stem cells (HSCs) and hematopoietic progenitor cells (HPCs) in the absence of active SARS-CoV-2 infection (10). Viral-vector and mRNA vaccines against SARS-CoV-2, may be engineered to compel the protein synthetic apparatus of recipient patients to synthesize the full-length SARS-CoV-2 spike glycoprotein in a non-throttleable manner (11). NATO and NATO-aligned countries, (such as Australia, Japan and Israel), have almost exclusively utilized viral-vector and mRNA vaccines, which forcibly compels the protein synthetic apparatus of recipient patients to engage in the selfsynthesis of the full-length SARS-CoV-2 spike glycoprotein (11). Conversely, non-NATO, nuclear capable nations such as: China, Russia, India, Pakistan, North Korea and Iran have utilized a variety of SARS-CoV-2 vaccines, including inactivated-virus vaccines, subunit vaccines and nucleotide vaccines which do not compel the self-synthesis of the full-length SARS-CoV-2 spike glycoproteins (11). This report examines the strategic sequelae resulting from the disparity in SARS-CoV-2 vaccination policies between NATO/NATO-aligned nations and non-NATO, nuclear-capable nations such as: China, Russia, North Korea and Iran, in the context of the doctrine of Combined-Arms Cyber Warfare.
\end{abstract}




\section{Overview}

Combined-Arms Cyber Warfare, also known as Sixth Generation Warfare, Total Information Warfare, Electronic Warfare, Blackout War and Non-Contact Warfare, represent innovative methods and means for conducting warfare against military forces as well as, the civilian populations and/or infrastructures which they defend ${ }_{(1)}$. Combined-Arms Cyber Warfare utilizes cyber-attack, sabotage of infrastructural elements and Electromagnetic Pulse (EMP) attack, alone or in combination with conventional military tactics ${ }_{(1)}$. Nuclear EMP attack has been acknowledged as a central component of the military doctrines, plans and exercises for Combined-Arms Cyber Warfare for the nations of Russia, China, North Korea and $\operatorname{Iran}_{(1)}$.

A Nuclear EMP attack consists of detonating a thermonuclear weapon in a manner that minimizes the energy delivered on-target, in the form of blast (overpressure) and thermal

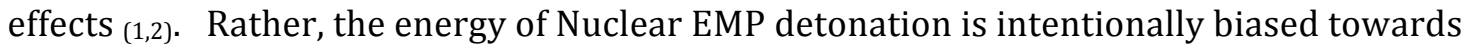
the release of prompt radiation, primarily in the form of gamma-neutron radiation (1). The tactical utility of Nuclear EMP, as harnessed by the doctrine of Combined-Arms Cyber Warfare, resides in the fact that Nuclear EMP weapons impart devastating electromagnetic effects on targeted areas (1).

Nuclear EMP attacks may consist of unmodified fission-fusion devices (thermonuclear weapons, a.k.a. "hydrogen bombs") detonated at altitudes high above a designated target, thus minimizing the ability of blast and thermal energy to reach the targeted area; but simultaneously enabling the saturation of the target area in prompt radiation emissions and a profoundly intense electric field ${ }_{(1)}$. In July 1962, the United States conducted Operation Fishbowl, detonation-Starfish Prime, during which a thermonuclear device was detonated at high altitude over the Pacific Ocean ${ }_{(1)}$. The detonation of the Fishbowl-Starfish Prime fission-fusion device unexpectedly resulted in the overloading and failure of the power grid in Honolulu Hawaii, 1,400 km from the site of detonation (1). In another aspect of Nuclear EMP development, fission-fusion devices have been modified and/or specifically designed and constructed to minimize the proportion of detonation energy yielded as overpressure and thermal outputs, while maximizing the percentage of energetic yield generated as prompt radiation $(1,2,3)$. The prompt radiation spectrum of Nuclear EMP weapon detonation is dominated by gamma-neutron emissions, but also includes alpha particle, beta particle and $\mathrm{x}$-ray emissions $(1,2,3)$.

Depending on the power of prompt radiation emissions as well as the strength of the associated electric field, Nuclear EMP attacks have been reported to be capable of "suppressing practically all classic electronic equipment...causing the melting or evaporation of metal in printed circuit boards or causing structural changes of electronic elements" (1). Nuclear EMP weapons include what are referred to as "Enhanced-EMP" and/or "Super-EMP" weapons, which are Nuclear EMP weapons purposely designed to maximize gamma-neutron emissions along with other prompt radiation components, so as to facilitate electric field generation, with strengths of the resultant electric fields registering from 10 to $200 \mathrm{kV} / \mathrm{m}_{(1)}$. Nuclear EMP generated electric fields are reported to have the ability to penetrate and melt any transistor and/or semi-conductor based electronic components (1).

Significant overlap exists between the design and function of "Enhanced-EMP/Super-EMP" weapons and that of Enhanced Radiation Weapons (ERW), colloquially known as "Neutron bombs" $(1,2,3)$. ERW are a class of fission-fusion devices that have been specifically designed 
to limit the energy of fission-fusion device detonation manifested as blast and thermal effects while maximizing the percentage of energetic yield generated as prompt gammaneutron emissions $(2,3)$. ERW development programs have originated from diverse strategic requirements, one such requirement was to develop a weapons system that could incapacitate large numbers of adversarial armored forces, which, were found to be operationally shielded from the blast and thermal effects of thermonuclear devices (3). ERW development programs were also driven by the need to quickly and with high reliability, disable sophisticated hypersonic and heavily shielded incoming thermonuclear warheads. (4). The W66 warhead is an example of such an ERW. Developed by the United States, the W66 was a component of the Sprint anti-ballistic missile system, where the primary effect of the W66 on incoming targeted warheads was destruction of the fissile core and electronic components via neutron-flux $(4)$.

Both Nuclear EMP weapons and ERW are fission-fusion devices, which feature specific design features and/or modifications such as, but not limited to: reductions in beryllium neutron reflectors and decreased device shielding, which have been found useful in limiting the energetic yield of detonation manifesting in the blast and thermal spectrums, while maximizing the energetic yield of detonation occupying the prompt radiation spectrum $(1,2,3,4)$. The prompt radiation spectrum of Nuclear EMP/ERW is dominated by gammaneutron radiation, and while gamma-neutron emissions comprise the majority of the prompt radiation spectrum emitted by Nuclear EMP/ERW, alpha particle, beta particle and $\mathrm{x}$-ray emissions also comprise prompt radiation emissions of Nuclear EMP/ERW detonation $(1,2,3,4)$.

ERW/Nuclear EMP design characteristics, such as a reduction of neutron reflectors and minimized device shielding, not only serve to minimize blast and thermal effects of detonation, while simultaneously maximizing prompt radiation emissions; said design characteristics also results in ERW/Nuclear EMP possessing smaller, lighter and less complex fission-fusion device design relative to standard thermonuclear warheads. The reduced physical dimensions of Nuclear EMP/ERW, relative to unmodified thermonuclear devices, enables a wide range of tactical deployments, such as, but not limited to delivery via hypersonic cruise missile, hypersonic boost-glide vehicle or an exo-atmospheric platform, such as a satellite or a sub-orbital vehicle, manned or unmanned $(1,4)$.

The prompt radiation spectrum of Nuclear EMP/ERW has been associated with the development of early transient incapacitation (ETI) among a wide range of vertebrate organisms (5). ETI is defined as an immediate decrement in the performance of a specified trainable task, as a result of the exposure to a supralethal dose of ionizing radiation, particularly high neutron content gamma-neutron radiation $(5,6)$. Furthermore, exposure to ionizing radiation, such as gamma-neutron radiation, at sufficiently high doses, leads to death from central nervous system (CNS) depression within hours to days $(5,6)$. Resultantly, it has been reported that non-human primates exposed to a $50 \mathrm{msec}$ pulse of high neutron content mixed gamma-neutron emissions, resulting in a dose of $4600 \mathrm{rad}$, caused: an immediate inability to perform trained tasks, with a significant majority (80\%) of subjects experiencing incapacitation within 24 hours of exposure, and a mean survival time following receipt of dose comprising a mere 37 hours (6). 
Neutrons have been reported to be more biologically hazardous than ionizing photons on a per dose basis (7). The pathological potency of neutron emissions is due in part to the fact that not only do neutrons directly induce biological damage, but neutrons also induce the release of three and four intensely ionizing alpha particles upon interacting with carbon and oxygen nuclei respectively (7). Additionally, in an urban environment, ionizing photons are more likely to be blocked by structural elements inherent to the urban setting, whereas neutrons are more likely to be "slowed" by urban terrain, resulting in a "hardening" and "thermalization" of the neutron spectrum (7). Neutron emissions secondary to the detonation of a Nuclear EMP/ERW within an urban environment can expect Fast neutrons (approximately $14 \mathrm{MeV}$ ) to become "hardened" and "thermalized" into Slow neutrons (100 eV to $0.5-1.0 \mathrm{MeV}$ ) and even Thermal neutrons (0.025 eV) (7). Reports examining chromosomal damage resulting from neutron emissions have established clearly delineated relationships between neutron energy and chromosomal damage per unit dose (7). Findings indicate that the degree of chromosomal damage increases with decreasing neutron energy over the range of 0.37 to $2.3 \mathrm{MeV}$ (7). Additionally, Thermal neutrons have been reported to possess a greater relative biological effectiveness (RBE) in respect to cell death, singlestrand DNA breakage and double-strand DNA breakage when compared to the RBE of an equivalent strength dose of gamma-radiation $(7,8)$.

SARS-CoV-2, the human $\beta$-coronavirus implicated as the causative agent in the COVID-19 pandemic, encodes four classes of structural proteins: spike glycoproteins, envelope proteins, membrane proteins, and nucleocapsid proteins ${ }_{(11)}$. The full-length spike glycoprotein of SARS-CoV-2 has been reported to have the ability to localize to the cellular nucleus (9). Nuclear and chromatin-bound compartments of cells examined during subcellular fraction analysis have demonstrated the presence of the full-length SARS-CoV-2 spike glycoprotein (9). Full-length SARS-CoV-2 spike glycoproteins, when localized to the nucleus, have been reported to directly inhibit double-strand break (DSB) DNA repair mechanisms (9). Full-length SARS-CoV-2, spike glycoprotein inhibition of DSB DNA repair is mediated via a pronounced inhibition of both the non-homologous end joining (NHEJ) repair and homologous recombination (HR) pathways ${ }_{(9)}$. Comparatively, the S1 and S2 subunit of the SARS-CoV-2 spike glycoprotein, demonstrate no significant inhibitory effect on DSB DNA repair in general, nor do the S1 and/or S2 subunits of the SARS-CoV-2 spike glycoprotein induce significant suppression of either the HR and/or NHEJ pathways (9). Additionally, reports also demonstrate that the full-length spike glycoprotein of SARS-CoV-2 impairs the expansion of hematopoietic stem cells (HSCs) and hematopoietic progenitor cells (HPCs) in the absence of active SARS-CoV-2 infection (10). Furthermore, HSCs and HPCs exposed to SARS-CoV-2 spike glycoprotein, in the absence of SARS-CoV-2 infection, were found to demonstrate reduced colony forming capacity (10).

SARS-CoV-2 has been the subject of the most intensive vaccine development effort in recorded history (11). The spectrum of SARS-CoV-2 vaccine candidates developed globally, demonstrates an expansive diversity in respect to mechanisms of action as well as targeted antigenic epitopes (11). SARS-CoV-2 vaccine candidates deployed globally include: inactivated-virus vaccines, peptide/protein subunit vaccines, live-attenuated vaccines and nucleotide vaccines (11). Nucleotide vaccines represent a novel approach to vaccination technology. Nucleotide vaccines introduce an engineered nucleotide template, either in the form of DNA or RNA, into a the cells of a recipient patient; where the nucleotide template then compels the protein synthetic apparatus of the recipient patient to self-synthesize the viral antigen encoded by the nucleotide (DNA or RNA) template (11). Viral-vector and mRNA SARS-CoV-2 vaccines are examples of nucleotide vaccines (11). Many nucleotide vaccines 
against SARS-CoV-2 introduce a nucleotide template into recipient patients that subsequently compels the protein synthetic apparatus of recipient patients to selfsynthesize the full-length SARS-CoV-2 spike glycoprotein ${ }_{(11,12)}$. However, SARS-CoV-2 nucleotide vaccines may also be engineered to introduce a nucleotide template that compels the self-synthesis of a SARS-CoV-2 antigen, that constitute only a portion of the full-length SARS-CoV-2 spike glycoprotein, such as the S1 subunit of the SARS-Cov-2 glycoprotein or the receptor binding domain (RBD) of the $\mathrm{S} 1$ subunit ${ }_{(11,19)}$.

Significant differences have been reported to exist regarding policies and mandates, pertaining to vaccination against SARS-CoV-2, between NATO member countries/NATOaligned countries (such as Australia, Japan, Israel, South Korea and New Zealand) and nonNATO countries, in particular, those non-NATO nations with nuclear weapons capabilities. Namely, non-NATO nations have employed inactivated-virus vaccines, as well as, subunit vaccines against SARS-CoV-2 to a much greater degree than NATO and NATO-aligned nations (11).

NATO and NATO-aligned countries have instituted policies regulating SARS-CoV-2 vaccination that have almost exclusively mandated the use of SARS-CoV-2 nucleotide vaccines that encoded for (and thus compel, the self-synthesis of) the full-length SARS-CoV2 spike glycoprotein antigen (11). In contrast, non-NATO countries such as China, Russia, Iran, India, North Korea and Pakistan, (which also possess nuclear weapons capability), have enacted policies toward SARS-CoV-2 vaccination which constitute a significantly more eclectic strategy (11). SARS-CoV-2 vaccinations utilized by China, Russia, Iran, India, North Korea and Pakistan notably include inactivated-virus vaccines, and peptide/protein subunit vaccines, neither of which compel the protein synthetic apparatus of recipient patients to self-synthesize SARS-CoV-2 antigens, let alone the full-length SARS-CoV-2 spike glycoprotein (11).

The administration of mRNA SARS-CoV-2 vaccines, encoding for the full-length SARS-CoV-2 spike glycoprotein, have demonstrated the presence of enduring levels of vaccine-delivered mRNA templates, for months after vaccination ${ }_{(12)}$. Additionally, vaccine-encoded, selfsynthesized full-length SARS-CoV-2 spike glycoprotein antigens have been detected within the tissues of individuals vaccinated with mRNA SARS-CoV-2 vaccines, for months following the last instance of vaccination ${ }_{(12)}$. These facts, combined with the demonstrated inhibitory effects of the full-length SARS-CoV-2 spike glycoprotein on DSB DNA repair, creates a scenario where recipients of SARS-CoV-2 nucleotide vaccines encoding the full-length SARSCoV-2 spike glycoprotein may demonstrate enhanced vulnerability to Nuclear EMP/ERW induced DSB DNA damage for months following the last dose of said SARS-CoV-2 vaccination and possibly longer $(9,10,11,12)$.

Given the stark differences that exist between NATO/NATO-affiliated nations and nonNATO, nuclear weapons capable nations, in respect to the vaccination their respective populations against SARS-CoV-2; significant potential advantages and disadvantages abound in the context of Combined-Arms Cyber Warfare ${ }_{(1,11)}$. This report examines the strategic and tactical implications of vulnerabilities issuing forth from SARS-CoV-2 vaccination policies employed by NATO/NATO-aligned nations, in respect to Nuclear EMP/ERW detonation. Additionally, the strategic and tactical implications of the eclectic SARS-CoV-2 vaccination policies employed by non-NATO, nuclear-capable nations are explored, in the context of Nuclear EMP/ERW detonation as utilized by the Combined-Arms Cyber Warfare doctrine. 


\section{Polices toward SARS-CoV-2 vaccination instituted by NATO and NATO-aligned nations have created potentially devastating vulnerabilities to high neutron content prompt radiation emissions released secondary to Nuclear EMP/ERW attacks}

On August 25, 2021 United States Secretary of Defense Lloyd J. Austin III issued a memorandum ordering the "full" vaccination of all members of the United States Armed Forces who fall under the authority of the U.S. Department of Defense (DoD) (13). While the memorandum issued by Secretary Austin included provisions that DoD personnel voluntarily vaccinated with a World Health Organization (WHO) approved vaccine would be considered "fully vaccinated", the DoD has relied entirely on Moderna's mRNA-1273, Pfizer/BioNTech's BNT-162b2 and Jansen's (Johnson \& Johnson) Ad26.COV2.S vaccines when administering voluntary vaccinations to of members of the United States Armed Forces $(11,13,14)$. In respect to those members of the United States Armed Forces who found themselves subject to receiving a mandatory SARS-CoV-2 vaccine, the DoD's stated position is that only SARS-CoV-2 vaccinations, which have received full licensure from the United States Food and Drug Administration (FDA), would be administered to DoD personnel pursuant to Secretary Austin's mandate (13). As of the writing of this report, only Pfizer/BioNTech's BNT-162b2 and Moderna's mRNA-1273 have received full FDA marketing approval, with mRNA-1273 having just recently received FDA approval. Therefore, the overwhelming majority of United States Armed Forces personnel, who were vaccinated against SARS-CoV-2 as a result of Secretary Austin's August 25, 2021 mandate, would have received the Pfizer/BioNTech's BNT-162b2 (Comirnaty) vaccine, a SARS-CoV-2 nucleotide vaccine that encodes the full-length SARS-CoV-2 spike glycoprotein $(11,13,14)$.

BNT-162b2, mRNA-1273 and Ad26.COV2.S all constitute SARS-CoV-2 nucleotide vaccines, which introduce a nucleotide template encoding for the full-length SARS-CoV-2 spike glycoprotein, where said nucleotide template compels the protein synthetic apparatus of a recipient patient to synthesize the full-length SARS-CoV-2 spike glycoprotein in a nonthrottleable manner ${ }_{(11)}$. That vaccine-encoded, full-length SARS-CoV-2 spike glycoproteins are produced in a non-throttleable manner, within the cells of recipient patients in response to vaccination with BNT-162b2, mRNA-1273 and Ad26.COV2.S, can reasonably be considered to potentially enhance likelihood that a non-zero quantity of self-synthesized, full-length spike glycoproteins will reach the nuclei of the cells of vaccinated individuals intact and cause some level of DSB DNA repair inhibition $(9,10,11,12)$.

Full-length SARS-CoV-2 spike glycoproteins have demonstrated the ability to reduce DSB DNA repair function by approximately $90 \%$ relative to controls, via suppression of HR and/or NHEJ pathways (9). Full-length SARS-CoV-2 spike glycoproteins, localized to the nucleus, have been found to directly inhibit the NHEJ repair pathway by markedly inhibiting the formation of 53BP1 foci, a key checkpoint protein for the NHEJ repair pathway (9). Fulllength SARS-CoV-2 spike glycoproteins, localized to the nucleus, have been found to directly inhibit the HR repair pathway by markedly inhibiting the formation of BRCA1 foci, a key checkpoint protein for the HR repair pathway (9). Additionally, full-length SARS-CoV-2 spike glycoproteins have demonstrated the ability to impair the expansion of HSCs and HPCs, as well as the ability to reduce the colony forming capacity among populations of HSCs and HPCs exposed to SARS-CoV-2 spike glycoproteins ${ }_{(10)}$. 
No inactivated-virus SARS-CoV-2 vaccines have been approved for administration in the United States, either under Emergency Use Authorizations (EUA) or for full FDA marketing approval. In fact, the FDA has blocked approval of BBV152/Covaxin, a WHO-approved, inactivated-virus vaccine against SARS-CoV-2 during attempted applications for both EUA and full FDA approval by U.S. based licensee Ocugen $(15,16)$. No NATO nations have granted full approval/authorization for the use of an inactivated-virus SARS-CoV-2 vaccine. Albania, North Macedonia, Montenegro, Hungary and Turkey constitute the only NATO nations that have granted emergency use authorization for the use of an inactivated-virus vaccine against SARS-CoV-2.

BBIBP-CorV (Sinopharm) is an inactivated-virus vaccine against SARS-CoV-2 ${ }_{(11)}$. China has granted BBIBP-CorV full approval, while Iran, North Korea, and Pakistan have approved BBIBP-CorV for EUA. However, North Macedonia, Montenegro and Hungary are the only NATO nations to have approved BBIBP-CorV for EUA.

CoronaVac (Sinovac Biotech) is an inactivated-virus vaccine, which has been granted full authorization by China and emergency use authorization by Pakistan (11). No NATO nations have granted CoronaVac full authorization, while Turkey, Hungary, Albania and North Macedonia have granted CoronaVac emergency use authorization.

BBV152/Covaxin is an inactivated-virus vaccine developed by Bharat Biotech and the Indian Council of Medical Research-National Institute of Virology (11). BBV152/Covaxin has been granted full approval by India and has been granted emergency use authorization by: Bahrain, Botswana, the Central African Republic, Comoros, Ethiopia, Iran, Mauritius, Myanmar, Nepal, Paraguay, Philippines, Trinidad and Tobago, Vietnam and Zimbabwe.

FAKHRAVAC is an inactivated-virus vaccine developed by Iran's Ministry of Defense and Iran has granted FAKHRAVAC emergency use authorization. The Chinese Academy of Medical Sciences COVID-19 vaccine is an inactivated-virus vaccine and has been granted emergency use authorization in China. COVIran Barekat is an inactivated-virus vaccine developed by Shifa Pharmed Industrial Co. of Iran and has been granted emergency use authorization in Iran. CoviVac is an inactivated-virus vaccine produced by the Chumakov Centre of the Russian Academy of Sciences and has been granted emergency use authorization by Russia, Belarus and Cambodia. Minhai COVID-19 vaccine is an inactivatedvirus vaccine developed by Minahi Biotechnology and Shenzhen Kangtai Biological Products Co. Ltd. and has been granted emergency use authorization by China and Indonesia. QazCovid-in (QazVac) is an inactivated-virus vaccine developed by the Research Instutute for Biological Safety Problems in Kazakhstan and has been granted emergency use authorization in Kazakhstan and Krygyzstan. Sinopharm WIBP is an inactivated-virus vaccine developed by Sinopharm and the Wuhan Institute of Biological Products and has been granted full approval by China and emergency use authorization in the UAE, Venezuela, the Philippines, Peru and NATO-member North Macedonia. Turkovac is an inactivated-virus vaccine developed by the Health Institutes of Turkey in conjunction with Erciyes University and has been granted emergency use authorization by NATO member Turkey.

Additional factors restricting the availability of the population of the United States to obtain SARS-CoV-2 vaccines, which are not comprised of SARS-CoV-2 nucleotide vaccines that encode for the full-length SARS-CoV-2 spike glycoprotein, include the fact that Novavax's NVX-CoV2373, a recombinant SARS-CoV-2 nanoparticle vaccine constructed from the full- 
length, wild-type SARS-CoV-2 spike glycoprotein, ( $5 \mu \mathrm{g} /$ dose), has only demonstrated limited availability via clinical trial enrollment, as the application for NVX-CoV2373's EUA has repeatedly faced delays (17).

BBV152/Covaxin and NVX-CoV2373 are examples of inactivated-virus vaccines and subunit vaccines, types of SARS-CoV-2 vaccines, which introduce a fixed amount of pre-formed, full-length SARS-CoV-2 spike glycoprotein, per dose, into the bodies of recipient patients ${ }_{(11)}$. An exceedingly low probability exists for the potential of preformed, full-length SARS-CoV-2 spike glycoproteins, introduced into a recipient patient via BBV152/Covaxin or NVXCoV2373, to reach the nucleus of a cell without undergoing enzymatic cleavage into component subunits, such as, but not limited to S1 and/or S2 (11). The negligible risk of complete, full-length SARS-CoV-2 spike glycoproteins being localized into the nuclei of cells of individuals vaccinated with vaccines, such as, but not limited to: BBV152/Covaxin and NVX-CoV2373, may reasonably be expected to cause no inhibition of DSB DNA repair pathways mediated by full-length SARS-CoV-2 spike glycoproteins $(9,11,12)$.

Due to the fact that the onset of ETI has been associated with profound histamine release, which occurs as a protective response to gamma-neutron-induced DNA damage, vaccines which compel the self-synthesis of the full-length SARS-CoV-2 spike glycoprotein may: 1) increase the severity of histamine release for a given level of neutron emissions over expected baseline responses, and thus increase the severity of ETI for a given dose of radiation exposure or 2) reduce the time to onset of ETI for a given dose of radiation exposure or 3) reduce the threshold dose of gamma-neutron emissions required for the onset of ETI to occur $(5,6,7,8,18)$.

Considering both the inhibition of DSB DNA repair and the myelosuppressive effects mediated by the full-length SARS-CoV-2 spike glycoprotein, in the context of the fact that BNT-162b2 delivered mRNA nucleotide templates, encoding the full-length SARS-CoV-2 spike glycoproteins as well as the full-length SARS-CoV-2 spike glycoproteins (whose synthesis is compelled by said templates), have demonstrated a durable presence of at least 60 days post vaccination, (as evidenced by detection within the germinal centers of lymph nodes of BNT-162b2a vaccinated patients); a reasonable concern exists for the potential of a significant enhancement in susceptibility to gamma-neutron emissions secondary to Nuclear EMP/ERW detonation $(5,6,7,8,9,10,11,12)$. Individuals vaccinated with SARS-CoV-2 nucleotide vaccines encoding for the full-length SARS-CoV-2 spike glycoprotein, including but not limited to: BNT-162b2a, mRNA-1273, Ad26.COV2.S and ChAdOx1 nCov-19 vaccine (AZD1222), fall into the category of individuals potentially possessing an increased vulnerability to prompt gamma-neutron emissions secondary to a Nuclear EMP/ERW attack $(5,6,7,8,9,10,11,12)$.

Given the profound contrast in SARS-CoV-2 vaccination strategy between that of NATO/NATO-aligned nations' near monolithic reliance on SARS-CoV-2 nucleotide vaccines which compel the self-synthesis of the full-length SARS-CoV-2 spike glycoprotein, and that of non-NATO, nuclear-capable nations' adoption and deployment of an eclectic array of SARS-CoV-2 vaccinations, many of which do not compel the self-synthesis of the full-length SARS-CoV-2 spike glycoprotein; a striking strategic inequity, in the context of potential vulnerability to the effects of Nuclear EMP/ERW detonation, seems to have developed along geopolitical lines. 
Non-NAT0, nuclear-capable, nations appear to hold a distinct strategic advantage, as a function of the reduced relative susceptibility of their populations to prompt gamma-neutron emissions occurring secondary to the detonation of Nuclear EMP/ERW in the context of Combined-Arms Cyber Warfare.

A tangible mechanistic model exists to conclude that non-NATO nuclear-capable nations may not have incurred the same degree of enhanced vulnerability to prompt-radiation induced injuries, such as but not limited to ETI, relative to NATO/NATO-aligned nations, as a result of the eclectic SARS-CoV-2 vaccination programs adopted by these non-NATO nations.

China, Russia and North Korea have not approved or authorized the use of the SARS-CoV-2 vaccines: BNT-162b2a, mRNA-1273, Ad26.COV2.S and AZD1222. Rather, China, Russia and North Korea have deployed an array of SARS-CoV-2 vaccines, which include inactivatedvirus vaccines, peptide-subunit vaccines and nucleotide vaccines. The segment of the populations of China, Russia and North Korea that received vaccinations which do not compel the self-synthesis of the full-length SARS-CoV-2 spike glycoprotein are unlikely to be susceptible to the inhibition of DSB DNA repair pathways, nor the myelosuppressive effects of vaccine-induced full-length SARS-CoV-2 spike glycoproteins.

As a result of the eclectic SARS-CoV-2 vaccination policies deployed by China, Russia and North Korea, pure statistical probability dictates that a significantly lower percentage of the vaccinated populations of these nations will suffer vaccine-induced vulnerability to promptradiation emissions of Nuclear EMP/ERW, particularly those resulting from gamma-neutron emissions. This lack of enhanced vulnerability to gamma-neutron emissions in vaccinated individuals would appear to be a stark contrast to the vast majority of the vaccinated populations of NATO/NATO-aligned nations. Nuclear EMP attack has been reported to feature prominently in the Combined-Arms Cyber Warfare strategy of "de-escalation" adopted by Russia, China and North Korea (1). The strategy of "de-escalation", in the context of Combined-Arms Cyber Warfare, describes a doctrine in which conflict with NATO/NATOaligned nations are intended to be won via the precise deployment and detonation of a limited number of Nuclear EMP/ERW detonations, constituting a type of "shock and awe" first-strike attack designed to subsequently forces NATO/NATO-aligned adversaries into submission $_{(1)}$.

Cyber-attack and sabotage with or without the execution of traditional military tactics, would likely accompany the nuclear detonations of a "de-escalation" attack, in order to cause maximal internal confusion, chaos and instability among NATO/NATO-aligned adversaries and to further enhance the likelihood of capitulation (1). In this regard, the profound potential for the inhibition of DSB DNA repair pathways, caused by SARS-CoV-2 nucleotide vaccines that compel the self-synthesis of the full-length SARS-CoV-2 spike glycoprotein, may serve as an ideal adjuvant to "de-escalation" attacks $(1,2,3,4,5,6,7,8,9,10,11,12)$. As previously mentioned, ETI is a phenomena that has been reported in a wide-range of vertebrate species, with non-human primates demonstrating an immediate loss of ability to perform trained-tasks, often followed by a loss of consciousness $(1,5,6)$. ETI is typically associated with exposure to a supra-lethal dose of high neutron content, mixed gammaneutron radiation $(5,6)$. ETI has been reported to be the result of a massive release of histamine as direct response to radiation-induced DNA damage, particularly in arterial endothelial cells $(5,6)$. The histamine bolus subsequently induces profound arterial dilation, hypo-perfusion of the CNS, 
and deterioration of cognitive and motor abilities, which precedes a loss of consciousness $(5,6)$. ETI has traditionally been associated with a supra-lethal dose of prompt radiation, such as a high neutron content, mixed gamma-neutron radiation pulse, delivered at 4600 $\mathrm{rad} /$ dose over $50 \mathrm{msec}(5,6)$.

However, in the context of DSB DNA repair mechanisms, whose function may be impaired up to $90 \%$ by the presence of full-length SARS-CoV-2 spike glycoproteins localized to the nucleus, a significantly lower dose of prompt radiation emissions may be sufficient to instigate severe DNA damage and trigger the release of a profound histamine bolus $(5,6,7,8,9)$. The relationship between the impairment of DSB DNA repair mechanisms and prompt radiation emissions, may yield a scenario where some individuals who have had DSB DNA repair mechanisms strongly inhibited by full-length SARS-CoV-2 spike glycoproteins, may develop ETI upon exposure to typically sub-lethal doses of gamma-neutron emissions.

The onset of ETI at sub-lethal doses of gamma-neutron emissions, would enhance the effectiveness of a "de-escalation" attack via multiple avenues, including but not limited to: 1) enhanced on-target effect, as confusion, chaos and instability within both military and civilian populations would dramatically increase, particularly as highly-skilled individuals demonstrated a deterioration in ability to perform trained tasks, prior to suffering incapacitation, (this would include physicians \& surgeons, pilots, power-plant operators, security forces, commanders, truck drivers, etc.) 2) Reduced requirements for the number of Nuclear EMP/ERW to be detonated, as the onset of ETI at sub-lethal doses would function as a "force-multiplier". 3) Reduced requirements for the kiloton yield of Nuclear EMP/ERW necessary for the conducting the attack, and thus enabling smaller physical dimensions of the fission-fusion devices, which thus enables a larger range of delivery vehicles, as well as, expanded options for deployment. 4) The ability to achieve strategic surprise, as the disproportionately increased susceptibility of adversarial forces to sub-lethal doses of gamma-neutron emissions would potentially afford an attacking force the ability to perform tactical operations involving the detonation of a Nuclear EMP/ERW at a range that traditionally would not be considered a threat, (secondary to: distance, altitude, terrain and/or cover), but as a result of increased susceptibility of the adversarial forces, would result in enemy incapacitation.

Russia has fielded Sputnik V, a SARS-CoV-2 nucleotide vaccine that utilizes a dualadenovirus vector to deliver a nucleotide template, which compels the self-synthesis of the full-length SARS-CoV-2 (11). Russia has also fielded EpiVacCorona and CoviVac, a sub-unit vaccine and an inactivated-virus vaccine respectively ${ }_{(11)}$. Should the intelligence apparatus of NATO/NATO-aligned nations be able to ascertain any potential for the fact that there was a deliberate effort to avoid vaccination of individuals in key strategic positions, both military and civilian, with Sputnik V in favor of either EpiVacCorona or CoviVac, it may not necessarily signal an awareness of the tactical advantage that could be gained from a "deescalation" attack with Nuclear EMP/ERW in the context of Combined-Arms Cyber Warfare, as it may be an attempt to avoid SARS-CoV-2 nucleotide vaccine class-specific side effects, such as but not limited to: myocarditis, thrombogensis and thromboembolism within key personnel and/or demographics (11).

However, regardless of the circumstances surrounding vaccine disbursement, Russian SARS-CoV-2 vaccine utilization has been divided between EpiVacCorona, CoviVac, and Sputnik V. Thus, the fact remains that the Russian population has been vaccinated with multiple vaccine options, with only Sputnik V comprising a vaccine which may confer 
significantly enhanced vulnerability to gamma-neutron emissions $(5,6,7,8,9,11)$. Therefore, relative to NATO/NATO-aligned nations (which have relied almost entirely on the administration of SARS-CoV-2 nucleotide vaccines that compel the self-synthesis of the fulllength SARS-CoV-2 spike glycoprotein), Russia could reasonably be expected to possess less vulnerability issuing forth from the biological effects of prompt gamma-neutron emissions, secondary to Nuclear EMP/ERW detonation.

The SARS-CoV-2 policies of China and North Korea seem to demonstrate a great reliance on inactivated-virus vaccines as BBIBP-CorV, CoronaVac, the Chinese Academy of Medical Sciences COVID-19 vaccine, the Minhai COVID-19 vaccine, and Sinopharm WIBP all represent inactivated-virus vaccines approved for use either under full or emergency approval. Additionally, China's initial attempt at developing an indigenous mRNA SARSCoV-2 nucleotide vaccine, ARCoV/Walvax COVID-19 vaccine, delivers an mRNA template that compels the self-synthesis of the receptor-binding domain (RBD) of the SARS-CoV-2 spike glycoprotein and not the full-length spike glycoprotein (19). While the RBD of the SARS-CoV-2 spike glycoprotein has been reported to be associated with a number of potentially deleterious side-effects, such as but not limited to, thrombogenesis, the isolated RBD is not associated with increased vulnerability to gamma-neutron emissions secondary to impaired DSB DNA repair pathways $(5,6,7,8,9,11)$.

As a function of the size of its population, both civilian and military, combined with the strategy of deploying SARS-CoV-2 vaccines among its population in a form which do not compel the self-synthesis of the full-length SARS-CoV-2 spike glycoprotein; China is clearly the least effected, nuclear-capable, nation in the world, with respect to a potential vulnerability of its population to gamma-neutron emissions secondary to SARS-CoV-2 vaccine-induced impairment of DSB DNA repair pathways. Relative to NATO/NATO-aligned nations and potentially even Russia, China holds a distinct and significant strategic advantage in the resilience of its population to a Nuclear EMP/ERW detonation. The reduced vulnerability of the Chinese population to prompt gamma-neutron emissions secondary to a Nuclear EMP/ERW detonation, particularly in comparison to the enhanced vulnerability that is likely to be demonstrated by the populations of NATO/NATO-aligned nations, means Chinese society would be less susceptible to the destabilizing effects of a "de-escalation" attack, in the context of Combined-Arms Cyber Warfare.

\section{Strategic and tactical adaptations NATO and NATO-aligned nations must consider in order to survive the potential of SARS-CoV-2 vaccine-induced enhanced population vulnerability to prompt gamma-neutron emissions resulting from the detonation of Nuclear EMP/ERW, in the context of Combined-Arms Cyber Warfare.}

Given the August 25, 2021 mandate issued by Secretary Austin, the entirety of the United States Armed Forces should be considered, for operational purposes, to be compromised in regard to their durability in the face of exposure to prompt gamma-neutron emissions $(1,2,3,5,6,7,8,9,9,10,11,12,13)$. As a result of this compromise and the vast distances with which the deleterious effects of Nuclear EMP/ERW detonation can be projected, it would be highly advised to avoid any prolonged concentration of U.S. Armed Forces at static geographical coordinates (1). It is advised that when possible, U.S. Armed Forces be as widely dispersed as possible, over vast distances, to avoid excessive loss of operational capability in the event of a Nuclear EMP/ERW detonation $(1,2,3,4,5,6,7,8,9,10,11,12)$. The recommendation for vast geographical distribution is just as applicable on U.S. soil as it is during foreign deployments, as Nuclear EMP/ERW detonation can be executed from extremely high 
altitude, via hypersonic delivery vehicle or via covert means such as a shipping container $(1,4)$.

A second, but painfully obvious strategic action, should be the immediate and unconditional prohibition of any further use of any and all SARS-CoV-2 nucleotide vaccines that compel the self-synthesis of the full-length SARS-CoV-2 spike glycoprotein, and where said vaccines include: Moderna's mRNA-1273, Pfizer/BioNTech's BNT-162b2, Jansen's (Johnson \& Johnson) Ad26.COV2.S vaccine and AZD1222 produced by Oxford-AstraZeneca $(11,13,14)$.

Detonation of a Nuclear EMP/ERW, in an urban setting, results gamma radiation particles, being blocked by the landscape more effectively than neutron emissions ${ }_{(7)}$. Furthermore, in an urban setting, Fast neutrons released during the detonation of a Nuclear EMP/ERW will "harden" and "thermalize", as said neutrons encounter the matter constituting an urban landscape (7). Slow and Thermal neutrons inflict more severe DNA damage with descending neutron energy levels (7). Therefore, the extra-corporeal absorption of Slow and Thermal neutrons is essential in order to minimize Nuclear EMP/ERW effects on vaccinated

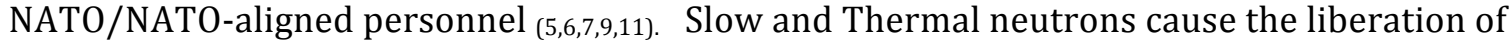
highly ionizing alpha particles when interactions with carbon and oxygen nuclei occur (7). When these interactions occur with the plentiful amounts of carbon and oxygen nuclei, which constitute the tissues of the human body, intense DNA damage occurs. Therefore, materials rich in oxygen, hydrogen, carbon and boron should be arranged as shielding in the environment of vaccinated NATO/NATO-aligned personnel.

Intermediate Bulk Containers (IBC) in the form of steel-caged, high-density polyethylene (HDPE) tote tanks filled with a solution of water and boric acid, may be stacked around the exterior and/or interior of barracks, hangars, hospitals and other locations at high risk of being subject to neutron bombardment secondary to Nuclear EMP/ERW detonation, in order to form a type of quickly deployable and transportable neutron shielding (20). Additionally, preventative dosing and/or fast-response anti-histamine therapy should be considered for vaccinated NATO/NATO-aligned personnel, particularly those individuals where a momentary lapse in task performance may have catastrophic consequences, such pilots.

Many questions exist regarding the enduring nature of SARS-CoV-2 nucleotide delivered nucleotide templates within the cells and tissues of vaccinated individuals, but at least a 60 day, post-vaccination interval has been established ${ }_{(12)}$. Additionally, questions exist regarding the enduring nature of vaccine-induced, self-synthesized full-length SARS-CoV-2 spike glycoprotein as well as the rate and/or magnitude with which these molecules are capable of localizing to the nucleus of a cell, thus impairing DSB DNA repair, however, a minimum of 60 -days post-vaccination and the capability to reduce DSB DNA repair by $90 \%$ have been demonstrated $(9,12)$.

Despite a multitude of topics requiring further scientific investigation, given the severity and finality of the Combined-Arms Cyber Warfare doctrine, (which advocates Nuclear EMP/ERW detonations as elements of "de-escalation" first-strike attacks), NATO and NATOaligned nations (which have deployed near-monolithic SARS-CoV-2 vaccination policies which administered SARS-CoV-2 nucleotide vaccines that compelled the self-synthesis of the full-length SARS-CoV-2 spike glycoproteins in a non-throttleable manner), must take immediate and purposeful actions, driven by the thoughts that the worst-case projections pertaining to enhanced vulnerability to gamma-neutron emissions in vaccinated individuals from NATO/NATO-aligned nations is known to be absolute fact. 
1) Peter Vincent Pry. Nuclear EMP attack scenarios and Combined-Arms Cyber Warfare. Report to the Commission to Assess the Threat to the United States from Electromagnetic Pulse (EMP) Attack. 2017 July

2) Glen I Reeves. Biophysics and medical effects of enhanced radiation weapons. Health Phys. 2012 Aug; 103(2):150-8. doi: 10.1097/HP.0b013e31824abef5

3) Fred M. Kaplan. Enhanced-Radiation Weapons. Sci. Am. 1978 May 01; 238:5. doi: 10,1038/scientificamerican0578-44

4) James Walker, Lewis Bernstein, Sharon Lang. Seize the High Ground: The U.S. Army in Space and Missile Defense. 2003 Center of Military History; U.S. Army Space and Missile Defense Command. Historical Office ISBN 0160723086

5) David Orlo Carpenter. Early Transient Incapacitation: A review with Consideration of Underlying Mechanisms. Armed Forces Radiobiology Research Institute. 1979 April. AFRRI-SR79-1

6) C. G. Franz, L. Clark, J. W. Cable. Primate Physical Activity Following Exposure to a Single 4600-Rad Pulsed Dose of Mixed Gamma-Neutron Radiation. Armed Forces Radiobiology Research Institute. 1976 August. AFRRI SR76-42

7) Daniela L. Stricklin, Jama VanHorne-Sealy, Carmen I. Rios, Lisa A. Scott Carnell, Lanyn P. Taliaferro. Neutron Radiobiology and Dosimetry. Radiation Res. 2021 May 1; 195(5):480-496. doi: 10.1667/RADE-20-00213.1

8) H. Maki, M. Saito, T Kobayashi, K. Kawai, M. Akaboshi. Cell inactivation and DNA single-and double-strand breaks in cultured mammalian cells irradiated by a thermal neutron beam. Int J Radiat Biol Relat Stud Phys Chem Med. 1986 Nov;50(5):795-809.doi: 10.1080/09553008614551231.

9) Hui Jiang, Ya-Fang Mei. SARS-CoV-2 Spike Impairs DNA Damage Repair and Inhibits V(D)J Recombination In Vitro. Viruses. 2021 Oct 13;13(10):2056. doi: $10.3390 / v 13102056$

10) James Ropa, Scott Cooper, Maegan L. Capitano, Wouter Van't Hof, Hal E. Broxmeyer. Human Hematopoietic Stem, Progenitor and Immune Cells Respond Ex Vivo to SARS-CoV-2 Spike Protein. Stem Cell Rev Rep. 2020 Oct 21: 1-13 doi: 10.1007/s12015-020-10056-z

11) James Sheehan. Vaccination of elite athletes against SARS-CoV-2 and broader implications for public health policy. 2022 January. OSF Preprints. doi: 10.31219/osf.io/z64gx

12) Katharina Roltgen, Sandra C.A. Nielsen, Oscar Silva, Benjamin A. Pinsky, Kari C. Nadeau, Scott D. Boyd, et al. Immune imprinting, breadth of variant recognition and germinal center response in human SARS-CoV-2 infection and vaccination. Cell January 24, 2022. doi: 10.1016/j.cell.2022.02.018

13) U.S. Department of Defense. 2022. Secretary of Defense Austin Issues Guidance for Mandatory Coronavirus Disease 2019 Vaccina. [online] https://www.defense.gov/News/Releases/Release/Article/2745742/secre tary-of-defense-austin-issues-guidance-for-mandatory-coronavirus-disease-2/

14) 2022 [online]https://www.defehttps://www.defense.gov/News/NewsStories/Article/Article/2626913/dla-ships-pfizer-vaccines-overseas-for-dodyouth/source/GovDelivery/nse.gov/

15) Desk, I. 2022. Here's Why FDA Rejected Covaxin's Emergency Use In America. [online] India.com Available at:https://www.india.com/news/india/reason-whyus-fda-rejected-covaxin-emergency-use-permission-bharat-biotech-ocugen-federaldrug-agency-latest-news-update-4731442/ 
16) FierceBiotech. 2022. Ocugen COVID shot will have to wait for US market as FDA slaps clinical hold on phase 3 trial. [online] Available at:https://www.fiercebiotech.com/biotech/fda-stops-ocugen-from-starting-phase3-trial-covid-19-vaccine-already-authorized-17

17) Endpoints News.2022. Novavax's vaccine rollout crawls amid months of broken promises-report.[online] Available at:https://endpts.com/novavaxs-vaccinerollout-crawls-amid-months-of-broken-promises-report/

18) A. Monti Hughes, ECC Pozzi, SI Thorp, P Curotto, VA Medina, DJ Martinel Lamas, et al. Histamine reduces boron neutron capture therapy-induced mucositis an oral precancer model. Oral Diseaes (2015) 21. 770-777 doi: 10.1111/odi.12346

19) Gui-Ling Chen, Xiao-Feng Li, Xia-Hong Dai, Nan Li, Meng-Li Cheng, Zhen Huang, et al. Safety and immunogenicity of the SARS-CoV-2 ARCoV mRNA vaccine in Chinese adults: a randomized, double-blind, placebo-controlled, phase 1 trial. Lancet Microbe. 2022 Jan 24. doi: 10.1016/S2666-5247(21)00280-9

20) P. Rinard. Neutron Interactions with Matter (1997) 